\title{
VARIACIÓN EN EL PATRÓN DE COLORACIÓN DE ABRONIA LYTHROCHILA (REPTILIA: ANGUIDAE) Y SU CONSERVACIÓN EN LA ESTACIÓN BIOLÓGICA SAN JOSÉ, SAN CRISTÓBAL DE LAS CASAS, CHIAPAS, MÉXICO
}

\section{VARIATION IN THE COLORATION PATTERN OF ABRONIA LYTHROCHILA (REPTILIA: ANGUIDAE) AND ITS CONSERVATION IN THE SAN JOSÉ BIOLOGICAL STATION, SAN CRISTÓBAL DE LAS CASAS, CHIAPAS, MEXICO}

\author{
J. MANUEL ARANDA COELLO ${ }^{1,2}$ \\ ${ }^{1}$ Centro para la Conservación e Investigación de la Biodiversidad de los Altos de Chiapas (CECIBACH), Secretaría de Medio \\ Ambiente e Historia Natural. Km. 76.9 carretera federal libre No. 190, Tuxtla Gutiérrez-San Cristóbal Zinacantán, Chiapas, \\ México.<m.aranda.coello@gmail.com> \\ ${ }^{2}$ Red Mesoamericana y del Caribe para la Conservación de Anfibios y Reptiles (MesoHerp).<m.aranda.coello@gmail.com> \\ *Autor de correspondencia:<m.aranda.coello@gmail.com>
}

Recibido: 07/10/2018; aceptado: 19/08/2019; publicado en línea: 20/09/2019

Editor responsable: Rogelio Cedeño Vázquez

Aranda-Coello, J. M. (2019) Variación en el patrón de coloración de Abronia lythrochila (Reptilia: Anguidae) y su conservación en la Estación Biológica San José, San Cristóbal de las Casas, Chiapas, México. Acta Zoológica Mexicana (nueva serie), 35, 1-7. https://doi.org/10.21829/azm.2019.3502087

RESUMEN. Se documenta la variación natural en los patrones de color en Abronia lythrochila y su conservación en la Estación Biológica San José, San Cristóbal de las Casas, Chiapas. México, con el fin de contribuir al conocimiento morfológico e historia natural de la especie.

Palabras clave: Altiplanicie de Chiapas; dragoncito de labios rojos; historia natural; variación morfológica

Aranda-Coello, J. M. (2019) Variation in the coloration pattern of Abronia lythrochila (Reptilia: Anguidae) and its conservation in the San José Biological Station, San Cristóbal de las Casas, Chiapas, Mexico. Acta Zoológica Mexicana (nueva serie), 35, 1-7. https://doi.org/10.21829/azm.2019.3502087

ABSTRACT. We document the variation in color pattern of Abronia lythrochila, and its conservation in the San José Biological Station, San Cristóbal de las Casas, Chiapas. México, with the purpose to contribute to the knowledge morphology and natural history of the species.

Key words: Highlands of Chiapas; red-lipped arboreal alligator lizard; natural history; morphological variation

El lagarto orejas de espina o Kix' Xikin en lengua Tzotzil (Abronia lythrochila) es una especie que actualmente se encuentra clasificada por la Unión Internacional para la Conservación de la Naturaleza (IUCN por sus siglas en inglés) como Least Concern (LC) y Amenazada por la NOM-059-2010 
(SEMARNAT 2010). Fue descrita por Smith y Álvarez del Toro (1963) con base en un holotipo hembra (UIMNH-51013) procedente de la localidad tipo referida como Nachij, entre Tuxtla Gutiérrez y San Cristóbal de Las Casas, Chiapas. El ejemplar fue colectado el 11 de julio de 1956 por Miguel Álvarez del Toro. Esta especie se distribuye en los municipios de San Andrés Larráinzar, Chenalhó, Pantelhó, Zinacantán, San Cristóbal de Las Casas, Teopisca, Amatenango del Valle y Las Rosas, en las montañas de la Altiplanicie de Chiapas (Müllerried, 1982), entre los 2,000 y 3,000 msnm (Campbell \& Frost, 1993; Torres et al., 2013). Habita únicamente en los bosques de pino-encino y ocupa las plantas epífitas (bromelias) como fuente de agua, refugio y alimento (Peterson \& Nieto-Montes de Oca, 1996; Cruz Ruiz et al., 2011; Aranda-Coello, 2011; Torres et al., 2013).

Históricamente A. lythrochila fue sinonimizada (o mal identificada) con A. ochoterenai, su historia taxonómica y nomenclatural comienza cuando Martín del Campo (1939: 357-9) encuentra un espécimen y lo describe como Gerrhonotus fimbriatus; posteriormente Tihen (1954: 22,24) describe unos especímenes apoyado por la descripción previa de Martín del Campo (1939) los cuales clasifica como A. ochoterenai; más tarde Smith y Álvarez del Toro (1962) describen al ejemplar encontrado el 11 de julio de 1956 por Miguel Álvarez del Toro, como A. ochoterenai comparándolo con los ejemplares que Tihen $(1954: 22,24)$ había descrito, debido a que los especímenes concordaban perfectamente bien con el diagnóstico, aunado a la carencia de material adecuado para realizar la comparación entre ambas especies (A. lythrochila y A. ochoterenai); posteriormente, Smith y Álvarez del Toro (1963) realizaron el diagnóstico correcto del especimen encontrado el 11 de julio de1956 por Miguel Álvarez del Toro y la describen como A. lythrochila, demostrando la existencia de una especie críptica asombrosamente similar y aparentemente en simpatría con A. ochoterenai. Esto derivó en que Álvarez del Toro (1973) corrigiera el nombre de la especie en la segunda edición de su libro Los Reptiles de Chiapas (Campbell \& Frost, 1993).

Smith y Álvarez del Toro (1963) describieron el color de A. lythrochila de la siguiente forma: color olivo verdoso por encima, en el dorso varias manchas oscuras irregulares, formando anillos en la cola; a lo largo del pliegue lateral, grandes manchas amarillo azufre; en la cabeza manchas marrón rojizas, entre el cuello y parte delantera del tronco presenta bandas oscuras; infralabiales rojo sangre; región gular blanquecina, así como toda la superficie ventral; región anal anaranjado rojizo e iris amarillento. Asimismo, Álvarez del Toro (1973) describió la coloración de A. lythrochila en su libro Los Reptiles de Chiapas de la siguiente forma: pardo amarillento con manchas irregulares café rojizo y otras manchas amarillo limón en los costados (siendo el color más frecuente para la especie). Con el avance tecnológico se ha incrementado el estudio morfológico, por medio del análisis y caracterización de imágenes a través del color con la "fotoidentificación", lo que ha tenido un alto impacto en los diferentes grupos naturales (Infante-Rivero, 2018) y debido a que existen pocos ejemplos documentados de variaciones en la coloración en lagartijas del género Abronia (Clause, 2016) y únicamente se sabe de posibles variaciones de color en pocos ejemplares de $A$. lythrochila (Campbell \& Frost, 1993; Langner, 2014) aquí se describe y documentan la variación en el color de una población de A. lythrochila, mediante la aplicación de la foto-identificación para mejorar la descripción de la coloración de la población que habita en la Estación Biológica San José.

Durante varias salidas de prospección herpetológica a lo largo cuatro años (2014 a 2018) en las 16 hectáreas de bosque de encino-pino de la Estación Biológica San José (EBSJ), la cual esta ubicada en un área montañosa entre los 2,350 y los 2,380 msnm en el Municipio de San Cristóbal de las Casas, Chiapas; entre las coordenadas geográficas extremas: $16^{\circ} 43^{\prime} 26^{\prime \prime} \mathrm{N}$ y $92^{\circ} 41^{\prime} 57^{\prime}$ ' W, $16^{\circ} 43^{\prime} 21^{\prime \prime} \mathrm{N} \mathrm{y} 92^{\circ} 42^{\prime} 03^{\prime \prime}$ $\mathrm{W}, 16^{\circ} 43^{\prime} 19^{\prime \prime} \mathrm{N}$ y $92^{\circ} 41^{\prime} 59^{\prime}$ ' W, $16^{\circ} 43^{\prime} 23^{\prime}$ ' N y $92^{\circ} 41^{\prime} 55^{\prime}$ 'W. Se detectó la presencia de A. lythrochila mediante observación directa en recorridos diurnos, como lo sugieren Aranda-Coello et al. (2012) en donde se realiza la búsqueda de la especie con un barrido continuo del tronco de los árboles hasta las ramas, bromelia por bromelia, con una cámara inalámbrica (Go-pro) conectada a un dispositivo móvil (en tiempo real). 
Para la descripción de la coloración, se revisaron y fotografiaron 16 ejemplares. A cada individuo se le tomó una fotografía de cuerpo entero, con una cámara digital Coolpix B500 (6MP 1/2.3" BSI CMOS Sensor NIKKOR f/3.0-6.5 ED Lens 22.5-900mm 40x Optical Zoom Lens, 80x Dynamic Zoom) y mediante su matiz (diferenciar un color de otro), se realizó la descripción in vivo de la variación de coloración de los especímenes (esto con la intención de poder garantizar que no hay modificación de la coloración natural presentada en los ejemplares fotografiados), y para aquellos colores difíciles de percibir se utilizó el apoyo de una paleta de colores del Atlas de colores de Küper (1996). En cuanto a la descripción, se utilizó el término "todo el cuerpo" para referirnos desde el dorso de la cabeza, tronco, extremidades y cola; después de la descripción de los ejemplares se realizó la liberación de los mismos en el sitio de captura dentro área de estudio.

Del total de ejemplares analizados, 15 fueron adultos (cinco hembras y 10 machos) y un joven (macho), con un promedio de longitud total de los adultos de $23.86 \mathrm{~cm}$ y un peso promedio de $37.84 \mathrm{~g}$, en los cuales se observaron las siguientes variaciones de coloración:

Ejemplar 1: coloración celeste-turquesa en todo el cuerpo con algunos puntos rojo-naranja en algunas escamas dorsales y las espinas supra-auriculares. Superficie ventral celeste con una franja color cobre a lo largo de los pliegues laterales (Fig. 1-A); ejemplar 2: coloración marrón como fondo en todo el cuerpo; franjas dispersas color negruzco y puntos rojos en todo el dorso; la superficie ventral café claro; la mitad posterior de la cola en color negro y la mitad anterior con mezclas de color marrón y negro (Fig. 1-B); ejemplar 3: coloración marfil en todo el cuerpo con mezclas de color ocre en la cabeza, en las escamas de la cabeza aparecen pequeñas líneas y puntos irregulares de color negro, dispersas hasta el cuello, el vientre es amarillo-huevo con pintas color cobre (Fig. 1-C); ejemplar 4: pardo-grisáceo en todo el cuerpo con pequeñas machas dispersas en la cabeza, dorso, patas y cola color crema, así como granate en la mitad del dorso y parte de la cola, el vientre es naranja-grisáceo (Fig. 1-D); ejemplar 5: fondo negro-mate en todo el cuerpo con grandes manchas irregulares en la cabeza, dorso y parte de la cola, el vientre es gris-oscuro con una franja rojiza (Fig. 1-E); ejemplar 6: negro-azulado metalizado iridiscente como fondo para todo el cuerpo, con pequeñas líneas olivo verdoso en las patas delanteras y en ambos costados del cuerpo; puntos dispersos marrón rojizo, diminutos sobre cabeza, dorso y cola (Fig. 1-F); ejemplar 7: gris-marrón como fondo para todo el cuerpo, con pequeñas manchas color rojizo en la cabeza, la cual presenta una coloración lavanda y esta se va difuminado hasta llegar al cuello, en las escamas de la cabeza aparecen pequeñas líneas y puntos irregulares negro dispersas hasta el cuello, el vientre al igual que el cuerpo de color gris (Fig. 1G); ejemplar 8: coloración amarillo oscuro-limón como fondo para todo en cuerpo, con franjas prolongadas color ocre desde la cabeza hasta la mitad del dorso, en las escamas de la cabeza aparecen pequeñas líneas y puntos irregulares negro dispersas hasta el cuello, el vientre es amarillo-limón (Fig. 1-H); ejemplar 9: coloración salmón en todo el cuerpo, el cual es oscuro en los costados y parte de la cola, en las escamas de la cabeza aparecen pequeñas líneas y puntos irregulares color negro, el vientre es del mismo color salmón que el cuerpo (Fig. 1-I); ejemplar 10: coloración café-claro como fondo en todo su cuerpo, la cabeza tiene tonos amarillo-limón con machas difusas anaranjado-rojizo en el dorso y los costados son pardo amarillento, el cual se va difuminado en la cola (Fig. 1-J); ejemplar 11: coloración rojizo-anaranjado como fondo para todo el cuerpo, la cabeza y parte de la cola tienen una coloración cobre-rojizo-claro, intensamente más fuerte en la cabeza hasta el cuello, el vientre es amarillo con puntos color cobre (Fig. 1-K); ejemplar 12: negromate en todo el cuerpo, con manchas irregulares color olivo-verdoso debajo de las espinas supra-auriculares y en los costados del cuerpo, algunas escamas dorsales y a lo largo del pliegue lateral tiene pequeños puntos rojizos, vientre negro-grisáceo con pintas color cobre (Fig. 1-L); ejemplar 13: blanco inmaculado como fondo para todo el cuerpo, con franjas irregulares negro-brillante en todo el cuerpo y pequeños puntos marrón-rojizos a lo largo de ambos pliegues laterales; en las escamas de la cabeza aparecen pequeñas líneas y puntos irregulares color negro, el vientre es negro con pintas blancas irregulares (Fig. 1-M); ejemplar 14: coloración café-olivo claro como fondo para todo el cuerpo, una línea color marrón rojizo presente desde la cabeza hasta la mitad del dorso y la cola tiene líneas irregulares del mismo color, el vientre es color café claro con una franja rojiza (Fig. 1-N); ejemplar 15: coloración café claro como fondo para todo el cuerpo, con pequeñas líneas oscuras en el dorso y manchas rojizas en ambos costados del cuerpo, la cola presenta 
pequeños pigmentos negros y en la cabeza aparecen pequeñas líneas y puntos irregulares también en negro, el vientre es blanco inmaculado (Fig. 1-O); por último, el ejemplar 16: coloración grisácea como fondo en todo el cuerpo, con pequeñas franjas irregulares negras que van del cuello hasta la cola, la cabeza y el dorso presentan manchas rojizas, así como pequeñas líneas y puntos irregulares en negro, el vientre es grisáceo (Fig. 1-P).

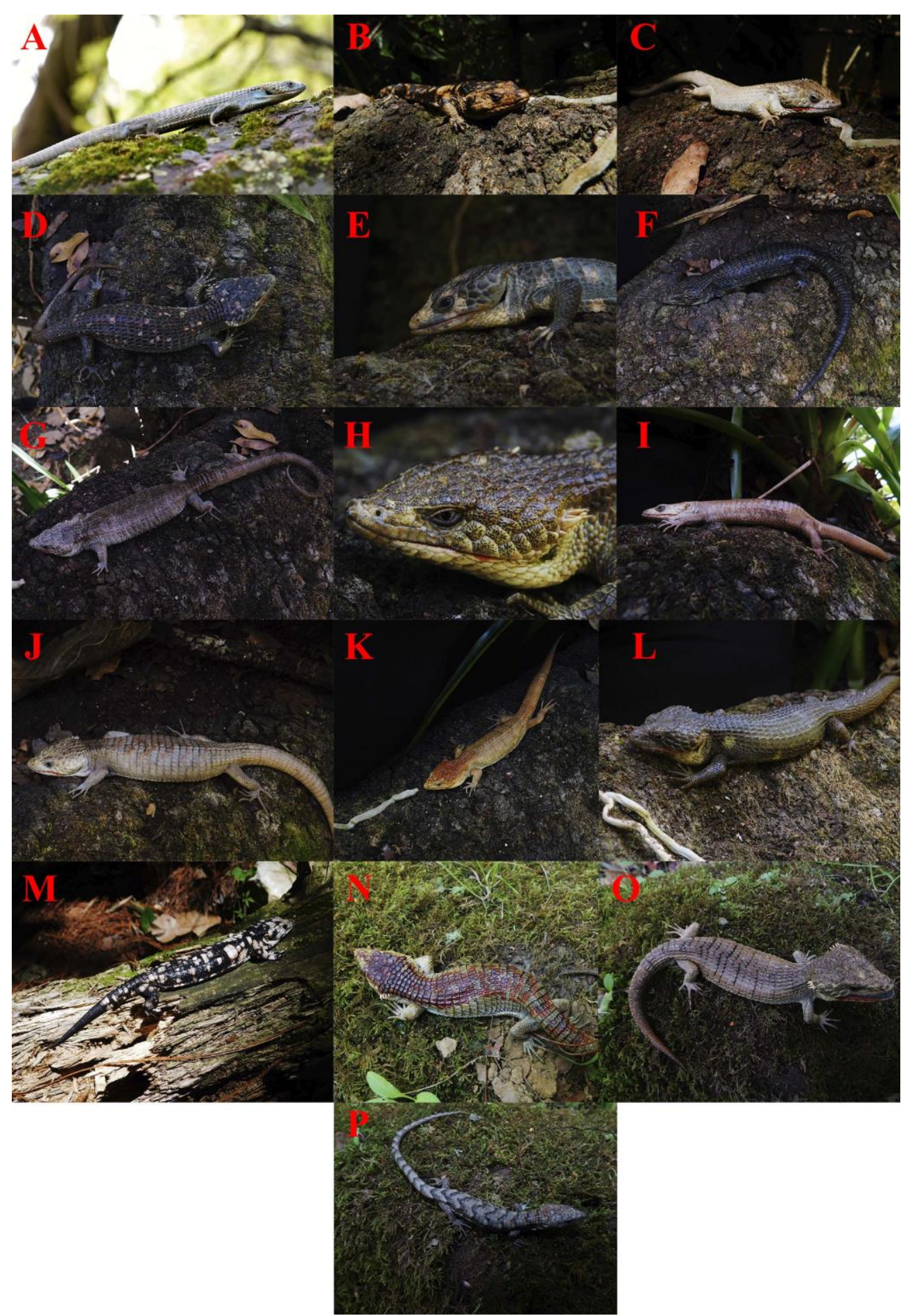

Figura 1. Primer plano, variación en el patrón de coloración en Abronia lythrochila. 
Con base en los resultados se encontraron nuevos patrones en la coloración de la especie, que no se han referido en la literatura (Smith \& Álvarez del Toro, 1963; Campbell \& Frost, 1993; Zaldívar Riverón et al., 2002; Langner, 2014), siendo estas: celeste-turquesa, ocre, salmón, grisáceo, marfil, granate, blancoinmaculado y cobre; asimismo se pudo identificar en los 16 ejemplares revisados, un total de \pm 24 mezclas de colores.

Zaldívar Riverón et al. (2002) describieron el patrón común de color blanco inmaculado en el vientre de A. lythrochila; sin embargo, la descripción y observación realizada en los ejemplares observados en este estudio muestra que el color del vientre en 15 ejemplares es similar al del resto del cuerpo y solo un ejemplar presentó el vientre de color blanco inmaculado. Por otra parte, se observó que en ejemplares con coloración café-claro, café-oliva, amarillo-limón, marfil y blanco, presentan en las escamas de la cabeza pequeñas líneas y puntos irregulares color negro, formando en ocasiones figuras parecidas a un asterisco.

Smith y Álvarez del Toro (1963) describieron el color de A. lythrochila como olivo verdoso por encima, en el dorso varias manchas oscuras irregulares, formando anillos en la cola; a lo largo del pliegue lateral, grandes manchas amarillo azufre; en la cabeza manchas marrón rojizas, entre el cuello y parte delantera del tronco presenta bandas oscuras; infralabiales rojo sangre; región gular blanquecina, así como toda la superficie ventral; región anal anaranjado rojizo e iris amarillento. Asimismo, Álvarez del Toro (1973) describió la coloración de A. lythrochila en su libro Los reptiles de Chiapas de la siguiente forma: pardo amarillento con manchas irregulares café rojizo y otras manchas amarillo limón en los costados. De los 16 ejemplares descritos en este trabajo, ninguno se apegó a las descripciones de color de dichos autores, por lo cual la descripción de los 16 ejemplares revisados representa nuevas aportaciones a la variación en la coloración de la especie (celeste-turquesa en todo el cuerpo con algunos puntos rojo-naranja en algunas escamas dorsales y las espinas supra-auriculares; marrón como fondo en todo el cuerpo; con franjas dispersas color negruzco y puntos rojos en todo el dorso; la mitad de la cola color negra; coloración marfil en todo el cuerpo con mezclas de color ocre en la cabeza; pardo-grisáceo en todo el cuerpo con pequeñas machas dispersas en la cabeza, dorso, patas y cola color crema; negro-mate en todo el cuerpo con grandes manchas irregulares en la cabeza, dorso y parte de la cola, por mencionar algunas); lo que no cambió en los 16 individuos revisados y que concuerda con la descripción original de la especie (Smith \& Álvarez del Toro, 1963) son los infralabiales rojo sangre y región anal anaranjado rojizo e iris amarillento.

Por otra parte, Campbell y Frost (1993) y Langner (2014) mencionan que los adultos de $A$. lythrochila tiene patrones de coloración que incluyen cuadros blancos y negros, grises con manchas rojas y un bronceado uniforme, lo que concuerda con dos de los individuos descritos en este trabajo: el ejemplar 13 que presentó cuadros blancos y negros (Fig. 1-M) y el ejemplar 16 que presentó un color gris con machas rojas (Fig. 1-P).

Dada la belleza de A. lythrochila y la variación en la coloración de la población analizada, existe un efecto negativo en la conservación de la especie en el área de estudio, ya que es objeto de tráfico ilegal de ejemplares por la demanda en el mercado de mascotas, con un valor de $\pm \$ 120$ peso mexicanos/ind. Los traficantes de vida silvestre realizan tratos con personas de comunidades indígenas cercanas para realizar la búsqueda, captura y extracción de individuos. Por desgracia, al ser organismos especializados en sus requerimientos biológicos, de no ofrecerles las condiciones óptimas en cautiverio llegan a experimentar inhibición de varios de sus procesos fisiológicos, alterando su complexión y coloración y en última instancia la muerte (obs. pers. Aranda-Coello).

Por otro lado, esta variación en la coloración los ha marcado como animales "malos", sobre todo aquellos que presentan una coloración oscura son lo más repudiados por los lugareños, ya que al ser encontrados son sacrificados. En la región de los Altos de Chiapas no se tiene ningún tipo de aprecio por estos animales, lo que constantemente pone en peligro la conservación de A. lythrochila (Aranda-Coello et al., 2012); aunado a las amenazas que ha tenido como la tala inmoderada en las zonas donde habita (Zaldívar 
Riverón et al., 2002) y a que actualmente es objeto de tráfico ilegal y sacrificio por las creencias de los lugareños (Aranda-Coello et al., 2012), además de la nula información sobre el tamaño poblacional y la fuerte destrucción y alteración que han sufrido en los últimos años los bosques de pino-encino y encino de la Altiplanicie de Chiapas (obs. pers. Aranda-Coello), debe considerarse la reclasificación de su categoría de riesgo en la IUCN.

A pesar de los esfuerzos de programas educativos ambientales como los realizados en los últimos cuatro años, desde el Centro para la Conservación e Investigación de la Biodiversidad de los Altos (antes Parque Zoológico y Estación Biológica San José) de la Secretaria de Medio Ambiente e Historia Natural de Chiapas, la desinformación que existe sobre la especie repercute en su conservación. Esperamos que en un futuro cercano A. lythrochila puede ser posicionada como un representante simbólico de los bosques de pino-encino y encino de la Altiplanicie de Chiapas, por su enigmática belleza y el beneficio ecológico que genera su presencia en estos ecosistemas.

\section{LITERATURA CITADA}

Álvarez del Toro, M. (1973) Los Reptiles de Chiapas. Segunda Edición. Tuxtla Gutiérrez. Chiapas, México, Gobierno del Estado. 178 pp.

Aranda-Coello, J. M. (2011) Evaluación de algunos efectos de la extracción tradicional de bromelias sobre la herpetofauna de los bosques de Chanal, Chiapas. Tesis de Licenciatura, Facultad de Ciencias Biológicas, Universidad de Ciencias y Artes de Chiapas, Tuxtla Gutiérrez, Chiapas, México. 38 pp.

Aranda-Coello, J. M., Ochoa-Ochoa, L. M., Naranjo-Piñera, E. J. (2012) Evaluación de algunos efectos de la extracción tradicional de bromelias sobre la herpetofauna de los bosques de Chanal, Chiapas. Acta Zoológica Mexicana (nueva serie), 28, 612-624.

Campbell, J. A., Frost, D. R. (1993) Anguid lizards of the genus Abronia: revisionary notes, descriptions of four new species, a phylogenetic analysis, and key. Bulletin of the American Museum of Natural History, 216, 1-121.

Clause A. G., Schmidt-Ballardo, W., Solano-Zavaleta, I., Jiménez-Velázquez, G., Heimes, P. (2016) Morphological variation and natural history in the enigmatic lizard clade Scopaeabronia (Squamata: Anguidae: Abronia). Herpetological Review, 47, 536-543.

Cruz-Ruiz, G. I., Mondragón, D., Santos-Moreno, A. (2011) The presence of Abronia oaxacae (Squamata: Anguidae) in tank bromeliads in temperate forests of Oaxaca, Mexico. Brazilian Journal of Biology, 72, 337-341.

Infante-Rivero, E. (2018) Variación en el patrón de coloración de Anablepsoides hartii (Cyprinodontiformes: Cynolebiidae) en arroyos costeros de Venezuela. Revista de Biología Tropical, 66, 293-302.

IUCN (2019) IUCN Red List categories and criteria. Version 3.1. IUCN, Gland, 32 pp.

Küppers, H. (1996) Atlas de los colores. Blume Ediciones. Barcelona. 162 pp.

Langner, C. (2014) Haltung und nachzucht der baumschleiche Abronia lythrochila. TERRARIA/Elaphem, $45,28-34$.

Müllerried, G. F. K. (1982) Geología de Chiapas. Segunda edición, Colección Libros de Chiapas, Serie Básica, Gobierno del Estado de Chiapas, Tuxtla Gutiérrez, Chiapas, México. 180 pp.

Peterson, A. T., Nieto-Montes de Oca, A. (1996) Sympatry in Abronia (Squamata: Anguidae) and the problem of Mario Del Toro Avilés' specimens. Journal of Herpetology, 30 (2), 260-262.

SEMARNAT (2010) Norma Oficial Mexicana NOM-059-SEMARNAT-2010, Protección ambiental Especies nativas de México de flora y fauna silvestres Categorías de riesgo y especificaciones para su inclusión, exclusión o cambio Lista de especies en riesgo. Diario Oficial de la Federación 30 diciembre, 2010.

Smith, H. M., Álvarez del Toro, M. (1963) Notulae herpetologicae chiapasiae IV. Herpetologica, 19, 100105. 
Smith, H. M., Álvarez del Toro, M. (1962) Notulae herpetologicae chiapasiae III. Herpetologica, 18, 101107.

Torres, M., Urbina, A., Vásquez-Almazán, C., Pierson, T., Ariano-Sánchez, D. (2013) Geographic distribution: Abronia lythrochila (red-lipped arboreal alligator lizard). Herpetological Review, 44, 624.

Tihen, J. A. (1954) Gerrhonotine lizards recently added to the American Museum collection, with further revisions of the genus Abronia. American Museum Novitates, 1687, 1-26.

Zaldívar Riverón, A., Schmidt, W., Heimes. P. (2002) Revisión de las categorías en el Proyecto de Norma Oficial Mexicana (PROYNOM-059-2000) para las especies de lagartijas de la familia Anguidae (Reptilia). Museo de Zoología "Alfonso L. Herrera". Departamento de Biología, Facultad de Ciencias, Universidad Nacional Autónoma de México. Bases de datos SNIB-CONABIO. Proyecto W026. México, D.F. 